\title{
ATIVIDADE ESPORTIVA PARA DEFICIENTES VISUAIS: UMA ANÁLISE QUANTITATIVA NO MUNICÍPIO DO RIO DE JANEIRO
}

\section{Sports activity for visually disabled people: a quantitative analysis in the city of Rio de Janeiro}

\section{Ramon Pereira de Souza, Darlan Campos}

\section{RESUMO}

A presente pesquisa investigou programas esportivos sociais de atividades físicas para deficientes visuais, no Município do Rio de Janeiro, sendo investigados quinze núcleos, que foram visitados ou com os quais houve uma comunicação à distância. O objetivo do estudo foi levantar o número de deficientes visuais atendidos pelos núcleos, segundo as Regiões Administrativas da Prefeitura da Cidade do Rio de Janeiro. A pesquisa descreve a importância do fomento da atividade física, para toda a população de deficientes visuais do Município, sendo os projetos esportivos sociais o palco primário de atletas olímpicos e paraolímpicos. Apesar da importância deste atendimento para esta clientela, a pesquisa concluiu que menos de $1 \%$ da população de deficientes visuais participa de programas de atividades esportivas. Recomenda-se um estudo similar em outras cidades brasileiras, dada a relevância dos projetos esportivos sociais para o futuro de atletas paraolímpicos.

Palavras-chave: Deficientes Visuais, Atividade Esportiva, Rio de Janeiro.

\section{ABSTRACT}

This research investigated social sporting programs of physical activities for visually disabled people in the city of Rio de Janeiro. Fifteen nucleuses were visited, or there was a communication at distance. The objective of the study is to note the number of visually disabled individuals attended by the nucleuses, according to the Administrative Regions of the City Hall of Rio de Janeiro. The research describes the importance of the physical activity for the whole population of visually disabled people of the city, being the social sporting projects the primary stage of Olympic and Paralympic athletes. Due to the importance of the sporting service for these customers, the study showed that less than $1 \%$ of the visually disabled population has access to it. A similar study is recommended in other Brazilian cities because of the relevance of social sporting projects for the future of Paralympic athletes.

Key words: Visually Disabled, Sports Activity, Rio de Janeiro.

Há um certo consenso de que atividades esportivas constituem uma das melhores formas de ocupar o tempo livre, mantendo o equilíbrio físico e mental, constituindo-se, portanto, em importante aliada para um estilo de vida mais ativo. Ter uma vida mais ativa não se resume em ter uma qualidade de vida melhor, que se traduz em aspectos como o estado de saúde, a longevidade, a satisfação no trabalho, as relações familiares ou a disposição para a vida ${ }^{(1)}$. No caso do deficiente visual, talvez sua maior importância

Universidade Salgado de Oliveira (UNIVERSO) - Rio de Janeiro - RJ - Brasil.

Revista de Educação Física 2008 Set; 142:50-57. Rio de Janeiro (RJ) - Brasil.

Recebido em 25.04.2008. Aceito em 09.06.2008. 
resida em dar-lhe condições de independência na questão de orientação e de mobilidade.

Pode-se definir qualidade de vida como um conjunto de parâmetros individuais, sócio-culturais e ambientais que caracterizam as condições em que vive o ser humano. $\mathrm{O}$ esporte, como manifestação cultural, destaca-se pelo envolvimento em atividades de lazer que, de diversas formas, contribuiriam para a melhoria do estado psicológico das pessoas envolvidas ${ }^{(2)}$. Seja pela ótica da inclusão social (3) ou da promoção da saúde ${ }^{(4)}$, a oportunidade da prática físico-desportiva, por todos, é amparada, na Constituição Federal, como um dos elementos essenciais para a melhoria da qualidade de vida da população.

Estudos comprovam que indivíduos portadores de deficiências se envolvem com atividades físicas e/ou desportivas desde a Grécia antiga ${ }^{(5)}$. No entanto, foi por volta de 1910 a 1920 que estas passam a ser sistematizadas, sobretudo em virtude da necessidade de programas de reabilitação para mutilados de guerra ${ }^{(6)}$. No que concerne, especificamente, ao desenvolvimento de atividades físicas para deficientes visuais, pode-se dizer que, em linhas gerais, não há muitas diferenças em relação às atividades para pessoas que enxergam. Na verdade, é necessário fazer ajustes em alguns procedimentos, organização e adaptações de certos jogos ou brincadeiras, a fim de oportunizar ao deficiente visual a prática de atividades desportivas.

Por outro lado, os deficientes visuais crescem a cada dia no Município do Rio de Janeiro. Segundo o censo de 2000, do IBGE (7), há uma população de 5.857.769 indivíduos na cidade, dos quais 828.399 são portadores de alguma deficiência e 519.348 são deficientes visuais (incapazes de ver - 6.300, com grande dificuldade de ver 65.493 e com alguma dificuldade permanente de ver 447.555). Em outras palavras, cerca de 71.793 , ou $1,2 \%$ de toda a população do Município, pode ser considerada deficiente visual.

Não é demais afirmar que um extrato populacional dessa magnitude precisa de serviços e estratégias de envolvimentos sociais, adaptadas e específicas. Tal não é diferente quando se trata de programas de atividades físicas. Esse é o principal foco do presente trabalho: há oferta de programas de atividades físicas, sejam elas de alto rendimento ou de lazer, para deficientes visuais, no Município do Rio de Janeiro?
Para responder este questionamento, foram levantados projetos esportivos sociais, desenvolvidos por instituições governamentais e não governamentais, no Município do Rio de Janeiro, que atendam deficientes visuais. Foram coletados dados, como o número de vagas para atendimento, analisando-se se tal oferta é compatível com o número de deficientes visuais da Região Administrativa. Além disso, foram detalhados, especialmente, o número de atendimentos oferecidos.

\section{$\mathrm{O}$ deficiente visual}

Um dos maiores entraves na integração do deficiente visual na sociedade, reside, na maioria das vezes, no ceticismo sobre sua verdadeira capacidade. É preciso uma postura de credibilidade frente a esta questão.

A definição da pessoa deficiente visual, segundo a Secretaria de Educação Especial, "é a perda ou redução total da capacidade de ver com o melhor olho e após a melhor correção ótica" (8). Em outras palavras, é a incapacidade total ou a diminuição da capacidade de ver, proveniente da imperfeição do sistema visual ou do próprio órgão da visão.

Para Tibola ${ }^{(9)}$, distingue-se dois tipos de deficiência visual: a cegueira e a visão subnormal (reduzida). Define-se cegueira como a perda total e/ou de resíduos mínimos de visão, levando o indivíduo a necessitar do "Sistema Braille" como meio de leitura e escrita, além de outros equipamentos específicos para o desenvolvimento educacional e integração social. Já a visão subnormal é caracterizada por a pessoa possuir um resíduo visual que a possibilita ler impressos a tinta, de forma ampliada, ou com o uso de equipamentos específicos.

Assim sendo, o deficiente visual (cego ou com visão subnormal) necessita de recursos didáticos diferenciados, para garantir sua possibilidade de desenvolvimento e sua participação nas atividades propostas. A Política Nacional de Educação Especial ${ }^{(8)}$ serve como fundamentação e orientação ao processo de educação de pessoas deficientes visuais, com condutas específicas, criando condições adequadas para o desenvolvimento de suas potencialidades, com vistas ao exercício consciente da cidadania.

\section{OBJETIVO}

O objetivo deste trabalho foi constatar quantos deficientes visuais são atendidos pelos núcleos esportivos sociais, seja em instituições governamentais ou em não 
TABELA 1

NÚMERO DE DEFICIENTES VISUAIS (DV), VAGAS POR REGIÃO

ADMINISTRATIVA EM PROJETOS DE ATIVIDADES FÍSICAS E RELAÇÃO ENTRE DEFICIENTES VISUAIS E VAGAS NOS PROJETOS.

\begin{tabular}{lccc}
\hline & Deficientes Visuais & Vagas & Relação DV/ vagas \\
\hline Área 1 & 3.419 & 24 & 142,45 \\
Área 2 & 9.954 & 428 & 23,25 \\
Área 3 & 30.555 & 52 & 587,59 \\
Área 4 & 6.185 & 0 & 6.185 \\
Área 5 & 21.680 & 122 & 177,7 \\
\hline
\end{tabular}

governamentais, segundo as Regiões Administrativas da Prefeitura do Rio de Janeiro.

\section{METODOLOGIA}

Foi utilizado o método quantitativo para investigar os projetos esportivos sociais que atendem deficientes visuais, em instituições governamentais e não governamentais, no Município do Rio de Janeiro. Foram analisados os regimentos internos ou projetos impressos, coletados diretamente nos locais ou pela internet.

As buscas de projetos esportivos e sociais do Município foram feitas através de endereços eletrônicos da Secretaria Municipal de Esporte e Lazer - SMEL, na Subsecretaria de Projetos Especiais, que administra todas as ações esportivas sociais para deficientes do Município do Rio de Janeiro. Através do endereço eletrônico do Ministério de Educação e Cultura, do Governo Federal, encontrou-se a listagem das Instituições Federais de Ensino, no Rio de Janeiro. O endereço das Instituições Municipais especializadas, no RJ, foi coletado junto à Secretaria de Educação do Município. Foram buscadas, ainda, informações no site do Ministério do Esporte, que divulga, em seu endereço eletrônico, todos os projetos sociais esportivos desenvolvidos em cada região do Brasil, com descrição das características de cada projeto e a abrangência de suas ações.

De posse dessas informações, passou-se a analisar o número de atendimentos declarados e em que medida isso supriria a demanda local de pessoas com deficiência visual. É importante ressaltar que o presente estudo não investigou atendimentos isolados, como aqueles encontrados em escolas, condomínios e associações, voltando sua atenção para projetos com maior impacto esportivo social. Ou seja, foram analisadas iniciativas que, de certo modo, poderiam ser entendidas como parte de estratégias de longo prazo, seja por estarem inseridas em políticas públicas, seja por traduzirem responsabilidade social de instituições privadas.

\section{RESULTADOS}

\section{Distribuição das vagas por Região Administrativa}

Para uma melhor visualização, a distribuição das Regiões Administrativas seguiu as recomendações da Prefeitura do Rio de Janeiro. Assim, cada área foi distribuída da seguinte forma:

Área 1: Portuária, Centro, Rio Comprido, São Cristóvão, Illha de Paquetá e Santa Tereza.

Área 2: Botafogo, Copacabana, Lagoa, Tijuca, Vila Isabel e Rocinha.

Área 3: Ramos, Penha, Irajá, Méier, Madureira, Inhaúma, Ilha do Governador, Anchieta, Pavuna, Complexo do Alemão, Maré e Jacarezinho.

Área 4: Barra da Tijuca, Jacarepaguá e Cidade de Deus.

Área 5: Bangu, Realengo, Campo Grande, Santa Cruz e Guaratiba.

A TABELA 1 mostra o número de deficientes visuais, o número de vagas e o número de deficientes visuais por vaga, em cada Área Administrativa. A TABELA 2, por sua vez, refina a informação, restringindo apenas aos sujeitos classificados como cegos.

De acordo com a TABELA 1, existe uma enorme defasagem entre o número de deficientes visuais e as 
TABELA 2

NÚMERO DE CEGOS, VAGAS POR REGIÃO ADMINISTRATIVA, EM PROJETOS

DE ATIVIDADES FÍSICAS E RELAÇÃO ENTRE DEFICIENTES VISUAIS E VAGAS

NOS PROJETOS.

\begin{tabular}{lccc}
\hline & Cegos & Vagas & Relação Cegos / vagas \\
\hline Área 1 & 316 & 24 & 13,16 \\
Área 2 & 1.035 & 428 & 2,41 \\
Área 3 & 2.778 & 52 & 53,42 \\
Área 4 & 685 & 0 & 685 \\
Área 5 & 1.486 & 122 & 12,18 \\
\hline
\end{tabular}

vagas ofertadas, em todas as Áreas Administrativas do Município do Rio de Janeiro. Restringindo o universo para cegos das mesmas regiões (TABELA 2), com número absoluto de cegos muito menor que o número absoluto de deficientes visuais, constatou-se que, também neste caso, em nenhuma das áreas o número de vagas foi suficiente para atendê-los.

\section{Projetos e instituições especializadas}

Como descrito antes, uma das preocupações deste estudo foi investigar todos os locais disponíveis para o atendimento esportivo de deficientes visuais. Os projetos relacionados eram, até o momento da coleta dos dados (dezembro de 2007), os que ofereciam oportunidade para esta clientela, no Município do Rio de Janeiro.

O número reduzido, bem como a falta de informações detalhadas quanto ao procedimento pedagógico aplicado, fez com que o estudo tivesse uma defasagem sobre as ações, no que tange o desenvolvimento esportivo social para deficientes visuais. Somente o Instituto Benjamin Constant atendia, especificamente, esta clientela, em todas as faixas etárias. Os outros dois projetos ofereciam vagas para todas as deficiências.

Este estudo contabilizou o número de deficientes visuais atendidos, em cada um deles, até dezembro de 2007, entendendo-se, como atendidos, os matriculados nas Secretarias dos Projetos ou das Instituições.

\section{_ Instituto Benjamin Constant}

Criado pelo Imperador D. Pedro II, através do Decreto Imperial $\mathrm{n}^{\circ}$ 1.428, de 12 de setembro de 1854, foi inaugurado no dia 17 de setembro do mesmo ano, com a presença do Imperador e da Imperatriz, com o nome de Imperial Instituto dos Meninos Cegos. Conta-se que o preconceito existente na época era muito grande e, com o aumento da demanda, foi idealizado e construído o prédio atual, que passou a ser utilizado a partir de 1890. Após a $1^{\text {a }}$ etapa da construção, recebeu o nome de Instituto Benjamin Constant (IBC), em homenagem ao seu terceiro diretor.

O IBC é considerado um Centro de Excelência Nacional, em questões de pesquisa, de atendimento e de desenvolvimento do deficiente visual. No IBC, são oferecidos cursos específicos que capacitam profissionais da área de educação, além de assessoria a outras instituições de ensino, bem como serviços como consultas oftalmológicas à população, reabilitação de pessoas com cegueira na fase adulta e atendimento de crianças deficientes visuais, desde a estimulação precoce até a nona série (ensino fundamental).

Em seu regimento, a Coordenação de Educação Física responde pelos atendimentos esportivos, atendendo todos os alunos, desde o ensino infantil até a reabilitação (pessoas que ficam cegas ou com baixa visão, na fase adulta).

Quando da coleta de dados, levantou-se que os alunos eram atendidos em duas sessões obrigatórias semanais, de 50 minutos cada, com opção de treinarem para as equipes representativas da instituição, em nível regional e nacional. Além disso, os alunos do IBC tinham a opção de participar das escolinhas esportivas, mantidas pela Coordenação com ajuda de estagiários e voluntários de Educação Física. Para os ex-alunos, eram desenvolvidos treinamentos nas áreas de atletismo, goalball, natação e 
futebol, com a perspectiva de disputar os campeonatos regionais e nacionais.

O grupo da reabilitação (os reabilitandos) era formado, em sua maioria, por pessoas que perderam a visão na fase adulta. A Coordenação de Educação Física do IBC oferecia atividades esportivas para esta clientela, como hidroginástica, musculação, natação e caminhada, com o objetivo de reintegrá-los na sociedade.

_ Programa Esportivo para Portadores de Deficiência (PEPPD)

Este é um projeto criado em 2002, com funcionamento nas áreas militares dos quartéis do Exército, no Município do Rio de Janeiro. Seus núcleos são localizados nos bairros da Urca, São Cristóvão, Realengo e Santa Cruz. O PEPPD estabeleceu, como meta primária, a capacitação de seu corpo técnico através de um curso de 30 horas, oferecendo noções básicas de cada deficiência atendida.

A atividade esportiva é desenvolvida com objetivo principal de incluir socialmente os seus usuários, melhorando a qualidade de vida. Seus objetivos específicos voltam-se para o desenvolvimento motor, para a melhoria dos aspectos afetivos e sociais, do relacionamento intrapessoal e interpessoal, além do desenvolvimento cognitivo.

Quando desta coleta de dados, eram atendidas 150 crianças deficientes, utilizando-se, como critério de seleção, as primeiras inscrições, que são substituídas de acordo com as ausências. Cada grupo possuía dois atendimentos semanais, com duas horas de duração cada, em dias alternados, sendo ofertadas atividades como futsal, goallball, natação, atletismo, ginástica olímpica, judô, capoeira e dança.

O grupo atendido era heterogêneo, envolvendo várias deficiências, com características regionais marcantes. No núcleo de Santa Cruz, por exemplo, a maioria dos alunos possuía algum tipo de deficiência mental e ou de autismo. $\mathrm{Na}$ Urca, havia um contingente maior de pessoas com deficiências mentais e físicas, enquanto que em Realengo e São Cristovão constatava-se uma forte presença de deficientes mentais e de portadores de Síndrome de Down. Os deficientes visuais, portanto, eram minoria entre os atendidos, em todos os núcleos.

Cada núcleo oferecia vagas para todas as deficiências, abrangendo deficientes físicos, mentais, paralisados cerebrais, autistas, bem como pessoas com outras síndromes. Com isso, apesar de não haver limitação quanto ao quantitativo de deficientes visuais que poderiam se inscrever, na prática, foi percebido um desinteresse desta população no atendimento. Além disso, neste programa, por não ser especificamente voltado para os deficientes visuais, não havia material adaptado para o desenvolvimento de atividades para estes alunos.

De acordo com o Coordenador Geral, quando do levantamento realizado por este estudo, eram atendidos 46 deficientes visuais em todos os núcleos, distribuídos assim: 15, no bairro da Urca; 11, em São Cristóvão; 07, em Santa Cruz; e 13, em Realengo.

\section{_ Vilas Olímpicas (VO)}

Desde 2001, a Secretaria Municipal de Esportes e Lazer (SMEL) começou a se preocupar com a questão da inclusão social das pessoas portadoras de deficiência, dentro das políticas públicas de esportes e lazer. Ao levantarmos os dados desta pesquisa, constatou-se que cada Vila Olímpica Municipal contava com uma equipe de profissionais, coordenando atividades específicas, além de realizarem o acompanhamento e o desenvolvimento dos alunos portadores de deficiência física.

Este programa de atendimento, pela Prefeitura, encontrava-se distribuído em dez núcleos no Município, sendo eles: Vila Olímpica Clara Nunes (Acari), Comendador Armindo da Fonseca (Campinho), Centro Esportivo Miécimo da Silva (Campo Grande), Vila Olímpica Oscar Schimidt (Santa Cruz), Vila Olímpica Mestre André (Padre Miguel), Vila Olímpica Gamboa (Centro), Vila Olímpica da Maré (Maré), Vila Olímpica Carlos Castilho (Complexo do Alemão), Vila Olímpica Jornalista Ary de Carvalho (Vila Kennedy) e Centro Integrado de Atenção à Pessoa Portadora de Deficiência - CIAD (Centro). Seu funcionamento era das terças às sextas-feiras e, de acordo com o projeto, o objetivo principal era educar e transformar valores equivocados da sociedade (como as noções de incapacidade e enfermidade) a respeito dos deficientes.

Dentre as atividades oferecidas, podem ser citadas o futsal, o basquete, a recreação, a ginástica rítmica, o xadrez, a capoeira e o handebol. A disponibilidade das informações, quanto ao número de deficientes visuais atendidos, não estavam discriminadas no site da Prefeitura do Rio de Janeiro e o acesso ao cadastro dos inscritos não foi permitido. Sendo assim, o presente estudo 
TABELA 3

NÚMERO DE DEFICIENTES VISUAIS POR NÚCLEO DAS VILAS OLÍMPICAS (VO) DA PREFEITURA DO RIO DE JANEIRO.

\begin{tabular}{lc}
\hline Núcleo e Local & $\begin{array}{c}\mathbf{N}^{\circ} \text { de } \\
\text { deficientes } \\
\text { visuais }\end{array}$ \\
\hline VO Clara Nunes - Acari & 18 \\
VO Comendador Armindo da Fonseca - & 21 \\
Campinho & \\
Centro Esportivo Miécimo da Silva - & 32 \\
Campo Grande & 11 \\
VO Oscar Schmidt - Santa Cruz & 22 \\
VO Mestre André - Padre Miguel & 12 \\
VO Gamboa - Centro & 16 \\
VO da Maré - Maré & 18 \\
VO Carlos Castilho - Complexo do & 185 \\
Alemão & \\
VO Jornalista Ary de Carvalho - Vila & 23 \\
Kennedy & \\
CIAD - Centro & \\
Total de deficientes atendidos no & \\
programa & \\
\hline
\end{tabular}

constatou o número de atendimentos a deficientes visuais, telefonando para cada coordenador de núcleo, conforme apresentado na TABELA 3, tendo sido percebida uma similaridade entre $o$ atendimento nas Vilas Olímpicas e o PEPPD, já que o atendimento é feito em conjunto, não havendo, portanto, a adoção de material didático adaptado para o deficiente visual, levando-o ao desinteresse.

\section{DISCUSSÃO}

Pode-se afirmar que esta é uma época em que a globalização exige, de todos os países, medidas que visem a humanização em várias dimensões da vida. Assim como o mercado de trabalho, aos poucos, vai se livrando de alguns preconceitos e destinando parte de suas vagas aos deficientes, a Educação Física também tem se preocupado com o atendimento desta população, em particular com os deficientes visuais. No que diz respeito ao Município do Rio de Janeiro, porém, os resultados obtidos neste estudo permitem pensar que isso esteja sendo feito de forma muito tímida e, talvez, pouco eficiente.
Por outro lado, a discussão dessas questões envolve aspectos importantes no diz respeito à promoção da saúde e à qualidade de vida, bem como à inclusão social de pessoas deficientes visuais. De forma abrangente, a qualidade de vida relaciona-se com o grau de satisfação do indivíduo com sua vida, em vários aspectos: moradia, transporte, alimentação, lazer, satisfação/realização profissional, vida sexual e amorosa, relacionamento com outras pessoas, liberdade, autonomia e segurança financeira ${ }^{(2)}$. Em outras palavras, a qualidade de vida remete à saúde emocional, influenciando, significativamente, na busca de um melhor padrão de vida, em um ciclo virtuoso.

A prática de atividades físicas pode contribuir com esse processo evolutivo, reforçando a auto-estima e a imagem corporal $^{(10)}$. Além disto, em uma dimensão mais concreta, contribui, decisivamente, com maiores níveis de mobilidade física e autonomia. Por certo, estes benefícios refletem nas relações de trabalho, na vida afetiva e na resolução dos problemas pessoais que surgem durante a vida.

De acordo com Araújo (11) e Guerra (12), tais aspectos assumem um significado ainda mais expressivo na pessoa deficiente visual. Os autores esclarecem que a falta do sentido da visão limita o ser humano em cerca de $85 \%$, na assimilação das informações que o cerca. Ao trabalhar atividades esportivas com uma pessoa deficiente visual, estar-se-á desenvolvendo os outros sentidos, permitindo o acompanhamento no seu desenvolvimento cronológico, assim como as obrigações que a sociedade impõe. 0 desenvolvimento da atividade física, em fase precoce, diferencia um deficiente visual, trabalhado desde a infância, de outro, não trabalhado, possibilitando ao primeiro uma integração menos sacrificada do que ao deficiente visual não trabalhado ${ }^{(10)}$.

Em suma, há uma concordância de que pessoas que participam de programas regulares de atividade física tendem a melhorar o seu auto-conceito e sua auto-estima, o que, em geral, resulta em um melhor relacionamento em todas as dimensões de suas vidas (trabalho, afetividade, saúde física, etc), proporcionando uma melhor qualidade de vida.

Infelizmente, os resultados apresentados constatam que as Políticas Públicas Municipais estão longe de oportunizar, a contento, a prática de atividades físicas aos deficientes visuais. No Município do Rio de Janeiro, o número de vagas oferecidas, em projetos esportivos 
sociais, para deficientes visuais, distribuída em suas Regiões Administrativas, não atinge nem 1\% da demanda. Esta é uma assustadora defasagem, havendo um evidente vácuo entre as obrigações do Estado e as necessidades da população de deficientes visuais, em flagrante conflito com direitos garantidos pela Constituição Federal ${ }^{(13,14)}$.

\section{CONCLUSÃO}

A partir das constatações apresentadas por este estudo, chega-se a duas conclusões: a primeira remete à pouca oportunidade da população de deficientes visuais, do Município do Rio de Janeiro, de se envolver em atividades físicas supervisionadas; e a segunda, que a população de deficientes visuais do Município do Rio de Janeiro não tem interesse em praticar atividade física.

Tanto uma, como outra conclusão, evidencia que o Município do Rio de Janeiro não oferece oportunidades para pessoas deficientes visuais no que tange à prática desportiva, negligenciando, neste aspecto, os direitos constitucionais desta população. Mesmo que não haja interesse das pessas portadoras deste tipo de deficiência, trata-se de obrigação do Estado educá-los para esta prática, levando-os a uma melhor qualidade de vida.

$\mathrm{O}$ número de vagas e atendimentos, em projetos e instituições oficiais, é sofrível (626), não alcançando $1 \%$ da população de deficientes visuais do Município. Mesmo naqueles locais em que estes atendimentos são possíveis, não existe uma material didático adaptado às suas necessidades, contribuindo ainda mais para afastá-los, limitando o seu desenvolvimento integral.

Ainda que no terreno da especulação, pode-se pensar que isso não seja muito diferente em outras cidades ou Unidades da Federação. Levantamentos adicionais desse tipo seriam desejáveis, contribuindo com a elaboração e com a implantação de políticas públicas efetivas, adaptadas para os deficientes visuais. Enfim, sugere-se que sejam realizados investimentos no desenvolvimento de estratégias didáticas específicas para o deficiente visual, o que tornaria mais fácil sua inclusão em programas de atividades físicas, de forma geral.

\section{REFERÊNCIAS BIBLIOGRÁFICAS}

1. Brancatti PR, Viana ES, Vilella RCA. Atividade motora adaptada para pessoas deficientes visuais. Londrina, Paraná: Ed. da Universidade Estadual de Londrina; 2001.

2. Minayo MCS, Hart ZMA, Buss PM. Debate - Qualidade de vida e saúde: um debate necessário. Revista Ciência e Saúde 2000; 5(1).

3. Glat R. Pesquisa em educação especial na pós-graduação. Série: questões atuais em educação especial - Vol VIII. Rio de Janeiro, RJ: Ed. Sette Letras; 1998.

4. Farinatti PTV. Promoção da saúde e educação física: conceito, princípios e aplicações. Rio de Janeiro, RJ: Ed. da Universidade do Estado do Rio de Janeiro; 2006.

5. ABRADECAR. Organização do desporto [on-line]. Disponível em: URL : http://www.abradecar.gov.br(01 jun. 2007).

6. Alencar B. Paraolimpíada - O Brasil no pódio. Rio de Janeiro, RJ: Comitê Paraolímpico Brasileiro; 1986.

7. Instituto Brasileiro de Geografia e Estatísticas (IBGE). Censo 2000. Dados estatísticos, armazém de dados. Tabela 870 [on-line]. Disponível em: URL: http://www.armazemdedados.rio.rj.gov.br/ (02 mai. 2007).

8. Brasil. Livro 1. Brasília, DF: Secretaria de Educação Especial, Política Nacional de Educação Especial - SEESP; 1994.

9. Tibola IM. Pessoa portadora de deficiência: integrar é o primeiro passo. Curitiba, Paraná: SEED; 1994.

10. Santos A. O corpo cego [on-line]. Disponível em: URL: http://www.ibc.gov.br (15 mai. 2007). 
11. Araujo PF. Desporto adaptado no Brasil: origem, institucionalização e atualidades. Campinas, SP: Ed. Universidade Estadual de Campinas; 1997.

12. Guerra JA. Orientação e mobilidade reabitam na cegueira. Revista Luis Braille 1999; jan/mar.

13. Brasil. Lei Federal no 8.069/90. Estatuto da Criança e do Adolescente - ECA. (13 ago.1990).

14. Brasil. Lei Federal, no 9.394. Lei de Diretrizes e Bases da Educação - LDB. (20 dez.1996).

\section{Endereço para correspondência:}

Rua Barata Ribeiro, 93 / 204, Copacabana Rio de Janeiro - RJ - Brasil

CEP 22011-001

Tel.: (21) 2530-2876 e-mail: futramon@gmail.com

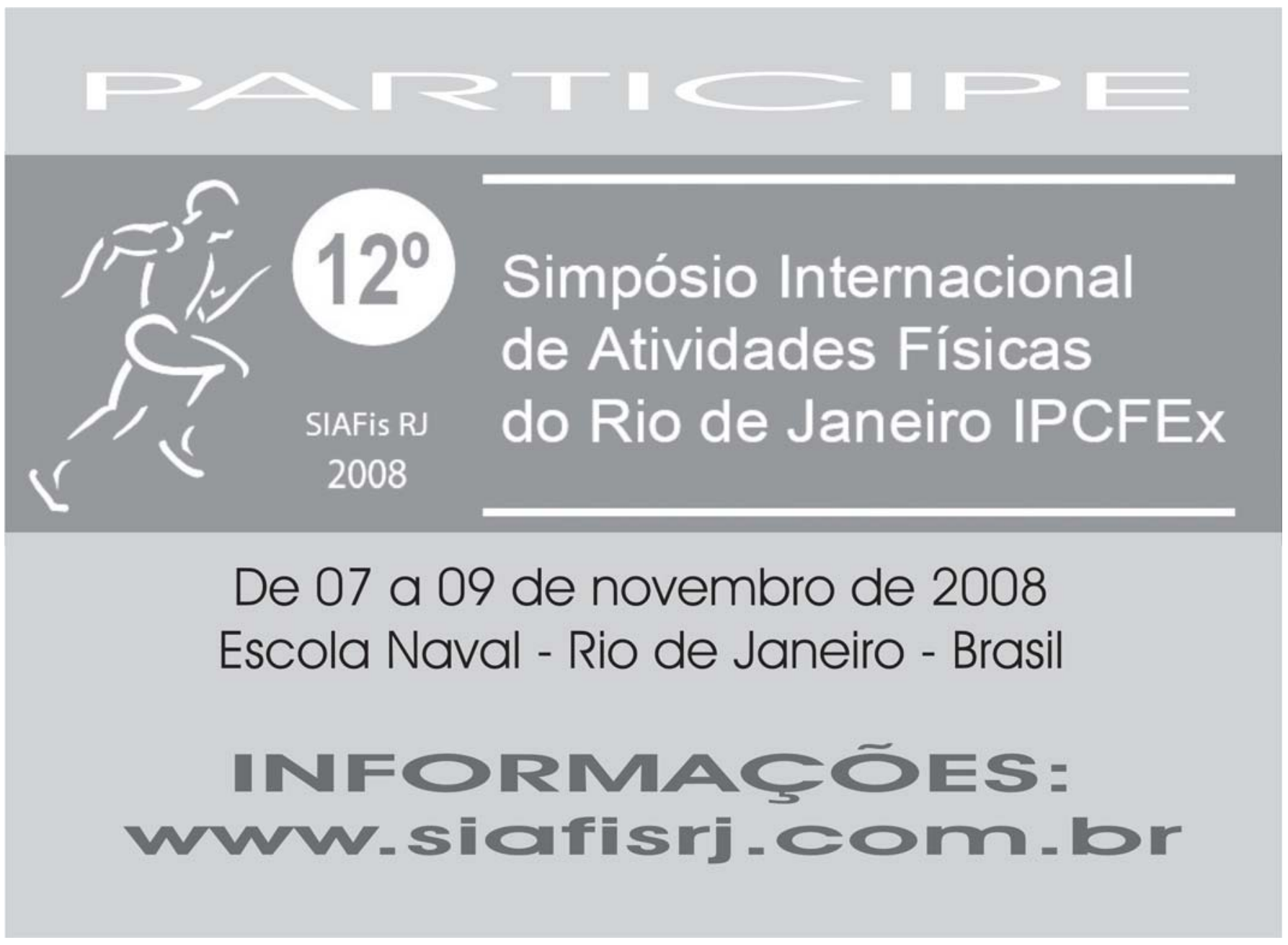

